\title{
Genetic Diversity of Chinese Bayberry (Myrica rubra Sieb. et Zucc.) Accessions Revealed by Amplified Fragment Length Polymorphism
}

\author{
Shuiming Zhang ${ }^{1}$, Zhongshan Gao, Changjie $\mathrm{Xu}^{2}$, and Kunsong Chen \\ Laboratory of Fruit Molecular Physiology and Biotechnology/The State \\ Agriculture Ministry Laboratory of Horticultural Plant Growth, Development \\ and Quality Improvement, Huajiachi Campus, Kaixuan Road 268, Zhejiang \\ University, Hangzhou 310029, China
}

\author{
Guoyun Wang \\ Yuyao Agricultural and Forestry Bureau, Yuyao 315400, China
}

Jintu Zheng

Extension Center for Forestry Science of Ningbo, Ningbo, Zhejiang 315010, China

\section{Ting Lu}

The People's Government of Zhangting Town, Yuyao 315410, China

Additional index words. amplified fragment length polymorphism (AFLP), Chinese bayberry (Myrica rubra Sieb. et Zucc.), genetic diversity

\begin{abstract}
Amplified fragment length polymorphism (AFLP) was used to analyze genetic diversity of 100 accessions of Chinese bayberry (Myrica rubra Sieb. et Zucc.), one of the widely cultivated fruit tree crops in southern China. Six E-NN/M-NNN primer combinations were selected and a total of 236 bands were obtained, of which 177 were

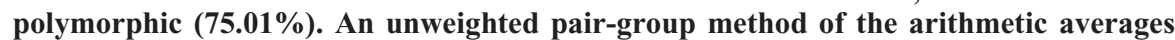
(UPGMA) was used to analyze the genetic relationships. The Dice's similarity coefficient among the Chinese bayberry accessions ranged from 0.75 to 1.00 and was 0.49 between Chinese bayberry and wax myrtle (M. cerifera L.). The 100 accessions of Chinese bayberry were clustered into two groups and seven subgroups. Subgrouping of Chinese bayberry was not related to the sex of the plant and color or size of the ripe fruit, but to some extent the region where the accession originated. However, the accessions from the same region did not necessarily belong to the same group or subgroup, which suggested the presence of extensive gene flow among different regions. Furthermore, close relationships between some morphologically similar accessions were found.
\end{abstract}

Chinese bayberry (Myrica rubra Sieb. et Zucc., Myricaceae) is a subtropical evergreen fruit tree native to China and other Asian countries that bears delicious, juicy fruit rich in healthy compounds (Chen et al., 2004, 2008; Zhang et al., 2008). In addition to being consumed fresh, various processed products, especially juice, are produced and some of them are available on overseas markets (Joyce, 2007; Karp, 2007). Today the crop

\footnotetext{
Received for publication 26 Sept. 2008. Accepted for publication $18 \mathrm{Dec} .2008$.

This work was supported by the Science and Technology Project of Zhejiang Province (2006C14016), the Ningbo Science and Technology Project (2005C100108), and the 111 project (B06014).

We thank Prof. Ian Ferguson, Dr. Andrew Allan, and Dr. Luud Gilissen for research advice and critical reading of this manuscript.

${ }^{1}$ Part-time PhD candidate from Key Laboratory of Pomology in Anhui Agricultural University, Hefei 230036, China.

${ }^{2}$ To whom reprint requests should be addressed; e-mail chjxu@zju.edu.cn.
}

has become one of the most important fruit tree crops in southern China with a production area reaching 865,000 acres, which even exceeds the area for citrus production in the United States (Karp, 2007). The fruit has gained international attention and acceptance in recent years for its unique appearance, flavor, and mouth feel (Joyce, 2007; Karp, 2007, 2008).

Chinese bayberry is a fruit with a long cultivation history of over 2000 years, but with only a three-decade-long research history. Its genetic diversity is far from being well investigated as compared with other main fruit crops in China. As many as 305 accessions were recorded with 268 among them being named as cultivars (Zhang and Miao, 1999). The cultivars were mostly classified on the basis of ripening date, fruit color, fruit weight, and stone characters, but there has not been consensus on cultivar classification (Chen et al., 2004). Occurrence of synonyms (a same cultivar with different names) as well as homonyms (different cultivars with the same name) are common. For example, the commercial production of this fruit crop frequently encountered difficulties in cultivar identification and discrimination. In addition, the degree of genetic diversity among the cultivars is poorly known.

Isozyme analysis (Handa and Kajiura, 1991), and later, random amplified polymorphic DNA (RAPD) (Lin et al., 1999), were applied for cultivar discrimination of Chinese bayberry. However, the isozyme patterns can be affected by factors such as developmental stage, whereas the RAPD results are generally of low repeatability as a result of the shortcomings of the technique. Such results need to be confirmed with other molecular marker techniques. Recently, intersimple sequence repeat (ISSR) was applied to investigate genetic relationships between Chinese bayberry accessions (Pan et al., 2008; Qian et al., 2006; Qiu et al., 2002; Xie et al., 2008), but these studies included relatively few genotypes (no more than 15 cultivars or lines).

In the present study, a collection of 100 Chinese bayberry accessions, 98 of them cultivars or lines, were analyzed by amplified fragment length polymorphism (AFLP), a reliable molecular marker with wide applications such as in parentage analysis, hybrid identification, cultivar discrimination, and genetic diversity studies (Heidi and Andrew, 2007). Through our data, we established the genetic relationships of commonly cultivated Chinese bayberry accessions, which can contribute to discrimination and sorting of cultivars and lines, evaluation and use of genetic resources, conservation of germplasm, and cross-breeding as well.

\section{Materials and Methods}

Plant materials. One hundred Chinese bayberry (Myrica rubra Sieb. et Zucc.) accessions and one wax myrtle (M. cerifera L.) from the China Bayberry Germplasm Repository, Yuyao, Zhejiang Province, China, were used in this study (Table 1). Ninety-nine Chinese bayberry accessions involved in this study were introduced from seven provinces of China (Fig. 1), and 71 of them were from Zhejiang Province, the largest producing area in China. In addition, one accession of Chinese bayberry and the wax myrtle were from Japan and the United States, respectively (Table 1). Young leaves were collected and subsequently frozen in liquid nitrogen and then stored at $-70{ }^{\circ} \mathrm{C}$ until use.

Analysis methods. Total genomic DNA was extracted from young leaves using an improved cetyltrimethylammonium bromide (CTAB) protocol. One gram of leaves was ground in liquid nitrogen to a fine powder before addition of $5 \mathrm{~mL}$ CTAB buffer $(2 \%, \mathrm{w} / \mathrm{v}$, CTAB, 100 m Tris- $\mathrm{HCl}, 50 \mathrm{~mm}$ EDTA, 1.4 $\mathrm{M} \mathrm{NaCl}, 0.5 \%$, v/v, $\beta$-mercaptoethanol, $\mathrm{pH}$ 8.0). The mixture was incubated at $65^{\circ} \mathrm{C}$ for 45 min followed by three extractions with an equal volume of chloroform/isopentanol $(24: 1)$. The supernatant was decanted and precipitated with cold isopropanol. The pellet was washed three times in $75 \%$ ethanol 
Table 1. List of 101 bayberry accessions included in this study. ${ }^{z}$

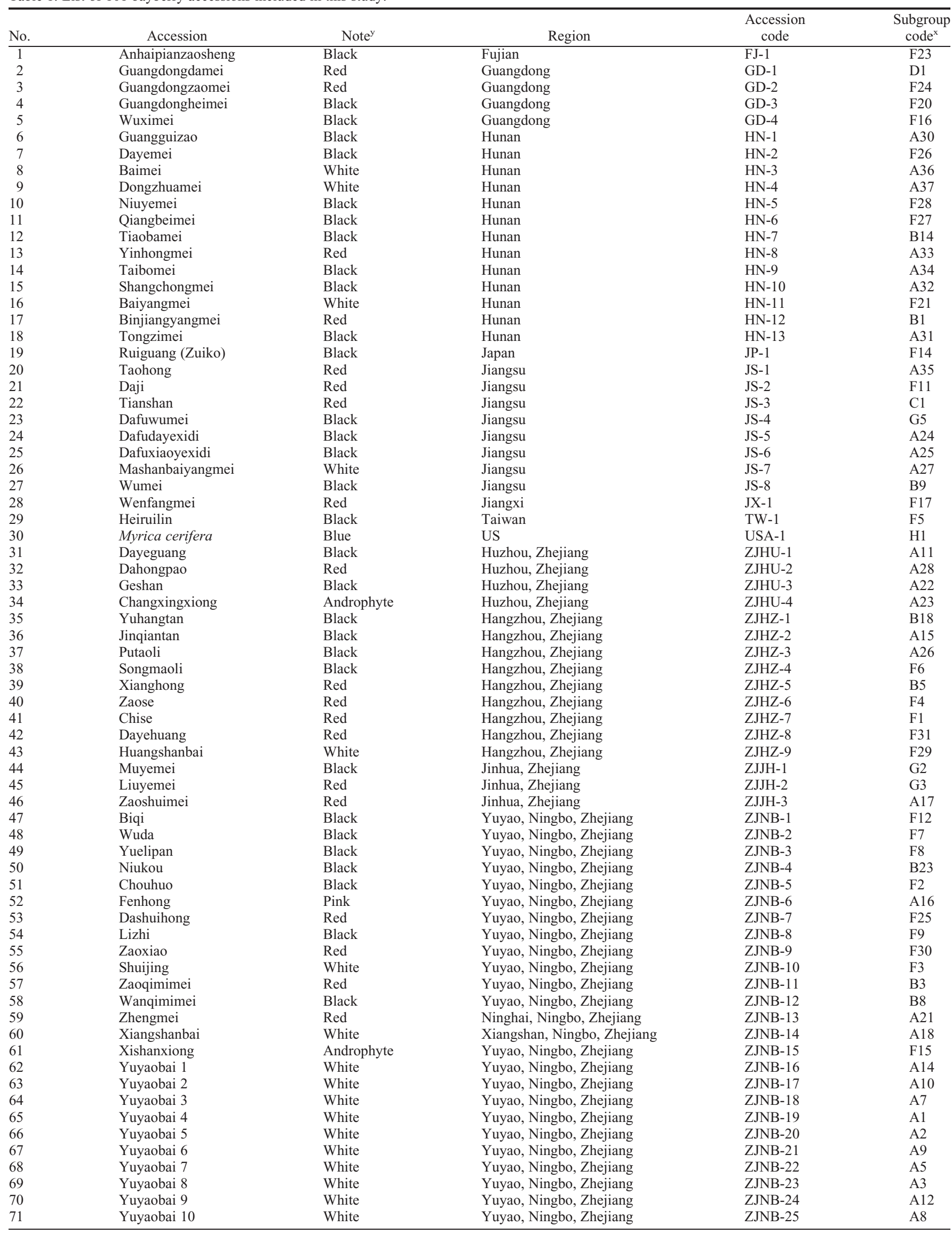


Table 1. (Continued) List of 101 bayberry accessions included in this study. ${ }^{2}$

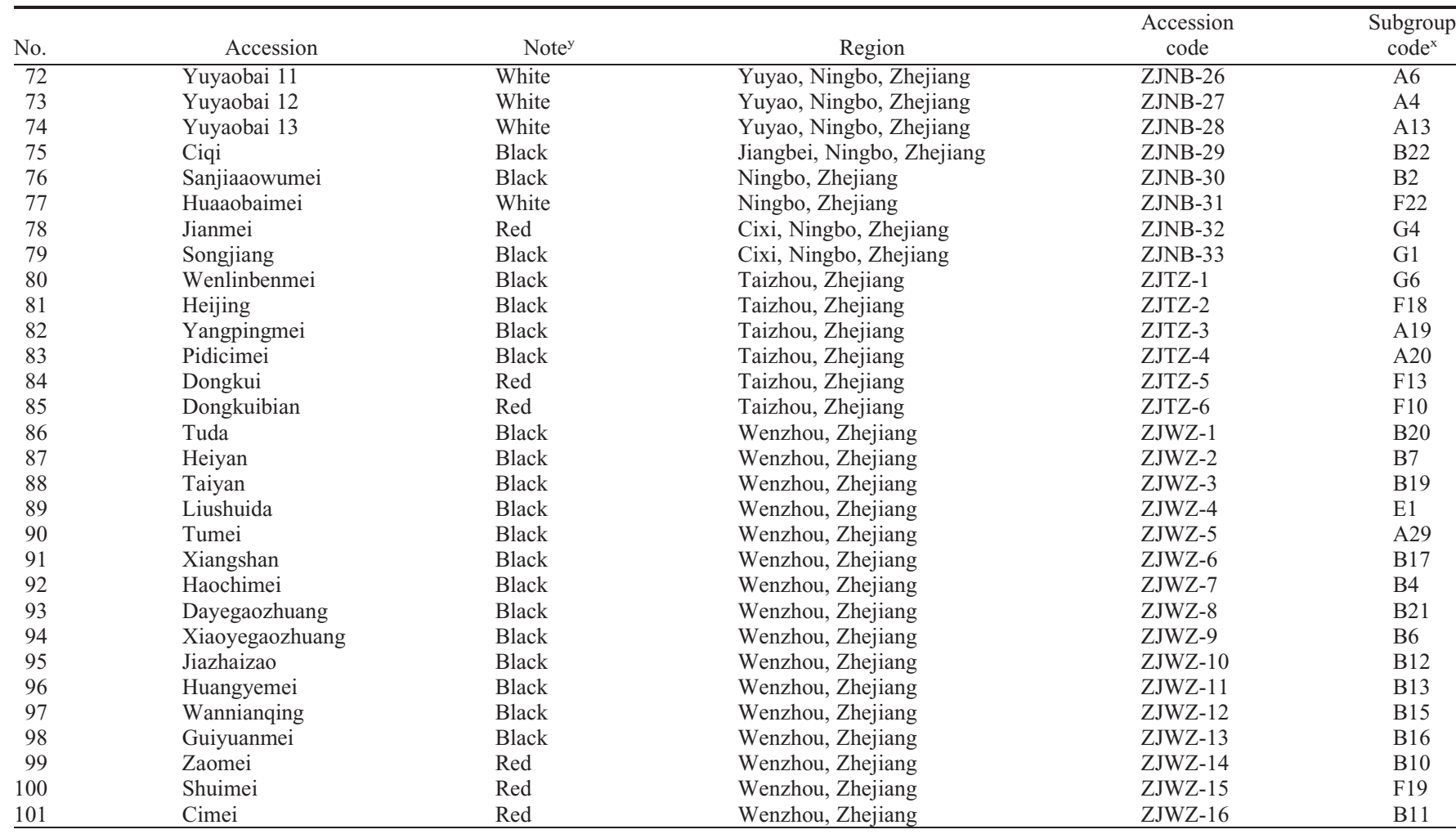

${ }^{\mathrm{z}}$ The accessions were sorted by the accession code.

${ }^{\mathrm{y}}$ Androphyte refers to androphyte accession, and the others indicate the color of mature fruit.

${ }^{x}$ Subgroup code was identical to those indicated in Figure 2.

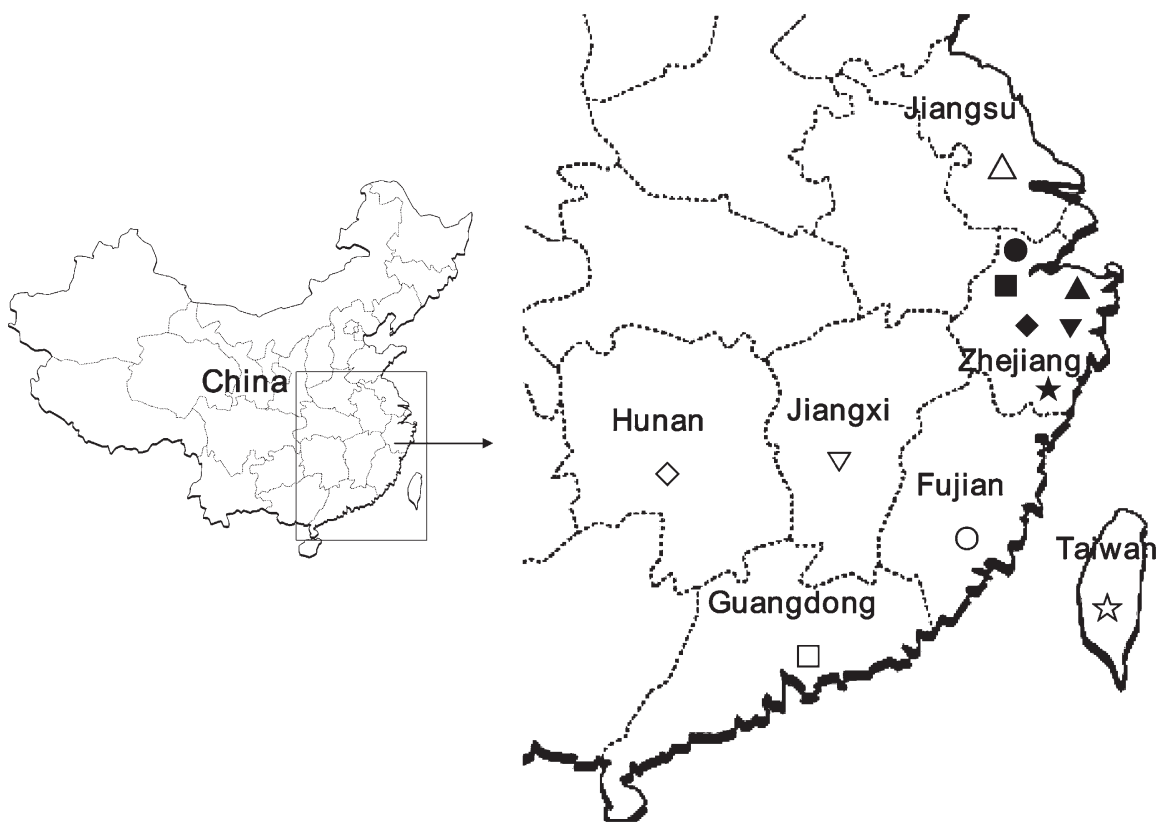

Fig. 1. Map of China indicating the region where the tree of the Chinese bayberry accessions originated. The symbols representing a province or a city in Zhejiang Province are the same as those indicated in Figure 2.

before being dissolved in TE buffer. RNA was removed by digestion with deoxyribonuclease-free ribonuclease A followed by chloroform/isopentanol extraction, ethanol precipitation, and finally the DNA pellet was redissolved in TE buffer. DNA concen- tration was quantified spectrophotometrically at $260 \mathrm{~nm}$ and was then adjusted to $100 \mathrm{ng} / \mu \mathrm{L}$.

AFLP was performed according to Vos et al. (1995) and Bao et al. (2008) except for the primers used. Restriction enzymes EcoRI and $M s e I$ were used. All polymerase chain reaction amplifications were repeated twice to ensure consistency. The amplified bands were scored as present (1) or absent (0) and only clear and unambiguous bands were considered valid. The percentages of polymorphic loci and Shannon's information index of the primer combination were calculated using the software package of POPGENE 1.31 (University of Alberta, Edmonton, Canada). Genetic similarity among all accessions was calculated according to Dice's similarity coefficients using the software NTSYS-pc 2.02 (Exeter Software, Setauket, NY). The dendrogram was constructed by FreeTree 0.9.1.50 (http://www. natur.cuni.cz/ $\sim$ flegr/programs/freetree.htm) using the unweighted pairgroup method with arithmetic averaging (UPGMA) option. The confidence of branch support was then evaluated by bootstrap analysis with 1000 replications. The dendrogram was printed using TreeView 1.6.1 (http://taxonomy.zoology. gla.ac.uk/rod/treeview.html).

\section{Results and Discussion}

Primer selection and amplification. Sixtyfour E-NN/M-NNN primer combinations were screened and six primer combinations were selected and applied to all accessions. The number of bands obtained with each primer combination ranged from 45 (E-AC/ $\mathrm{M}-\mathrm{CGC}$ ) to 34 (E-AA/M-CAA). With these six selected primer combinations, a total of 236 bands were amplified from 101 accessions, 


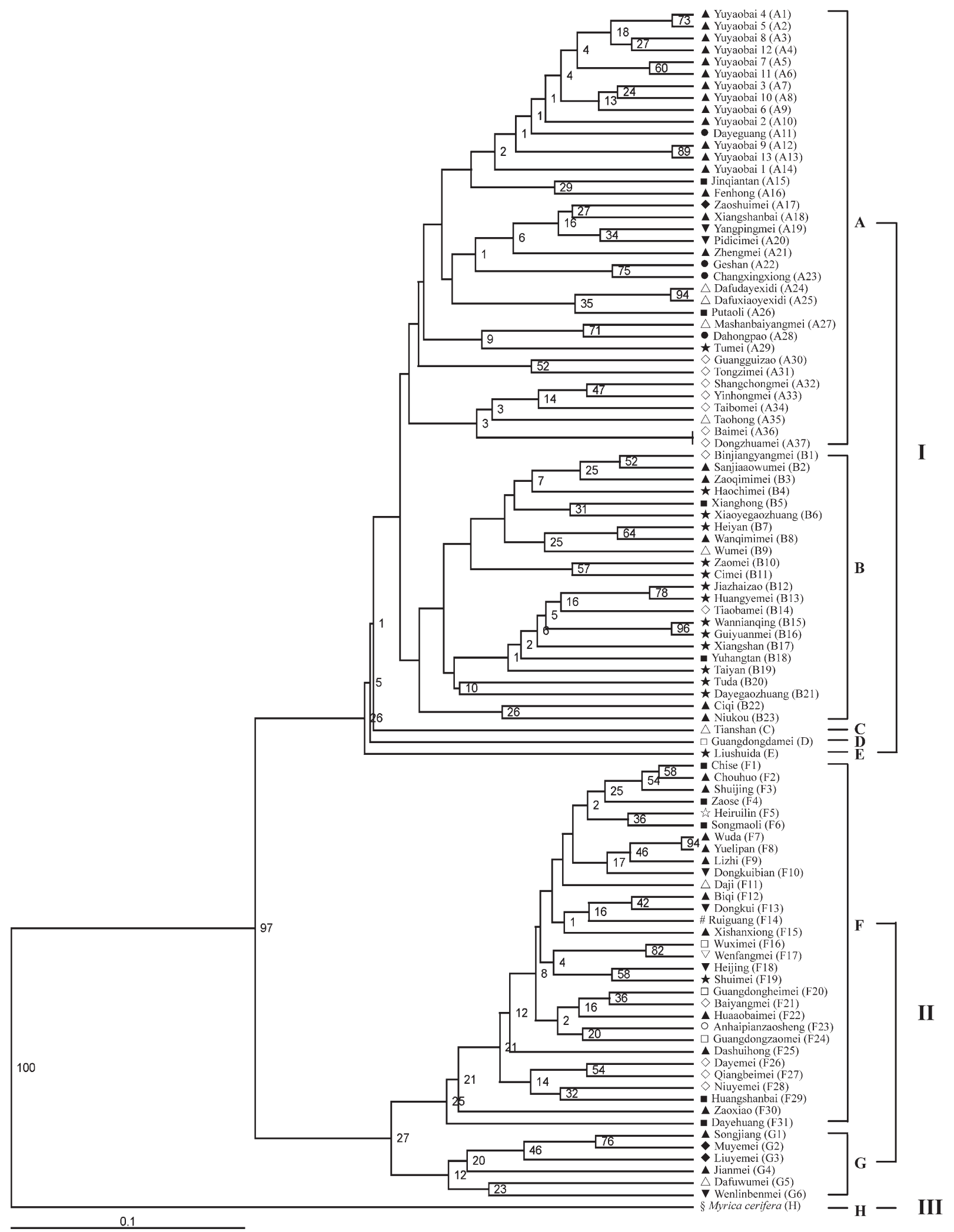

Fig. 2. Dendrogram of 101 accessions of bayberry resulting from the unweighted pair-group method of the arithmetic averages cluster analysis based on Dice's similarity coefficients after a 1000 replicate bootstrapping. Bootstrap values (in percent) are shown on the nodes. The branch lengths reflect the genetic distances between the accessions. The scale indicates a genetic distance of 0.1 units. The group codes, included in parentheses, were generated and used in Table 1 to facilitate reading. The symbols before the accession codes indicate the region where the accession originated, with $\bigcirc, \square, \diamond, \Delta, \nabla, i\}$ representing Fujian, Guangdong, Hunan, Jiangsu, Jiangxi, and Taiwan Provinces, and $\bullet, \boldsymbol{\bullet}, \boldsymbol{\bullet}, \mathbf{\Delta}, \mathbf{\nabla}$, and $\star$ for Huzhou, Hangzhou, Jinhua, Ningbo, Taizhou, Wenzhou city of Zhejiang Province, as shown in Figure 1, and \# and § for Japan and the United States, respectively.

and 177 of them were polymorphic. The percentage of polymorphic bands ranged from $64.86 \%$ (E-AC/M-CAT) to $86.67 \%(\mathrm{E}-\mathrm{AC} /$
M-CGC) with an average of $75.01 \%$. The average value of Shannon's information index was 0.2940 .
Genetic relationships among Chinese bayberry accessions. The lowest similarity coefficient, estimated by Dice's coefficient, 
was 0.49 between species of $M$. rubra and $M$. cerifera. Similar results were also reported previously with RAPD analysis (Lin et al., 1999). The highest similarity was $100 \%$ observed between Baimei (A36, indicating subgroup code, same as subsequently) and Dongzhuamei (A37), two accessions from Hunan Province with a white fruit color, suggesting that they are synonyms (Fig. 2). Among most Chinese bayberry accessions, the similarity coefficients ranged from 0.75 to 0.90 .

Based on the Dice's similarity coefficient, a dendrogram was constructed using the UPGMA clustering procedure, and the 100 Chinese bayberry accessions could be clearly subdivided into two groups with five and two subgroups for each group, respectively (Fig. 2). ISSR analysis also clustered Chinese bayberry accessions into two groups (Pan et al., 2008; Xie et al., 2008), but not as clear as the clustering was for AFLP data. Furthermore, subgrouping of Chinese bayberry was not possible in these two studies because of the limited number of accessions involved.

Subgroup A included 26 accessions from Zhejiang, seven accessions from Hunan, and four accessions from Jiangsu. All 13 accessions with a white fruit color from Yuyao, Ningbo, Zhejiang Province (A1 to A10, A12 to A14) were closely clustered in this subgroup, suggesting narrow diversity of these accessions. Subgroup B included 20 accessions from Zhejiang, two accessions from Hunan, and one accession from Jiangsu. Interestingly, 13 accessions from Wenzhou, Zhejiang Province, were clustered in this subgroup, which accounted for more than half of the accessions included. Subgroup F included 30 accessions from all seven provinces of China involved in this study and one accession from Japan. Using isozyme analysis, Handa and Kajiura (1991) indicated that this Japanese accession, 'Ruiguangmei' (F14), also known as 'Zuiko', was introduced from China. The data obtained in this study supported this assertion. Accession 'Heiruilin' (F5), a cultivar from Taiwan Province, was also clustered in this subgroup and showed a close relationship with some accessions from Zhejiang Province. Subgroup F consisted of a high number of commercially important cultivars, including 'Biqi' (F12) and 'Dongkui' (F13), the two most widely sold cultivars.

The clustering results indicated that accessions from the same geographic region did not necessarily belong to the same genetic group. For instance, the 37 accessions in Group II covered all seven provinces involved in this study, suggesting that extensive gene flow must have taken place among these regions. However, connections between the genetic and geographical distribution of Chinese bayberry were also observed. For example, all four accessions from Huzhou City of Zhejiang Province appeared in Subgroup A, 13 of 16 accessions from Wenzhou City of Zhejiang Province in Subgroup B, and three of four accessions from Guangdong Province in Subgroup F. All except for one accession in Group I were from Zhejiang, Jiangsu, or Hunan Province only, suggesting that the gene flow occurred more frequently among these three provinces. Qian et al. (2006) made similar conclusions through analysis of 14 Chinese bayberry accessions originated in Zhejiang and Jiangsu Province by using ISSR.

There is no sign of clustering related to sex of the plant and color or size of ripe fruit. Two androphyte accessions involved in this study, 'Changxingxiong' (A23) and 'Xishanxiong' (F15), appeared in Subgroups A and F, respectively. Coincidently, the 21 accessions with white fruit color appeared in either Subgroup A or Subgroup F. 'Dongkui' (F13), a cultivar bearing the biggest fruits, with an average fruit weight of $\approx 20$ to $25 \mathrm{~g}$, was most closely related to 'Biqi' (F12) with average fruit weight of $\approx 9$ to $10 \mathrm{~g}$.

The 13 Yuyaobai accessions (A1 to A10, A12 to A14) and 'Shuijing' (F3) appeared in different subgroups, although all of them originated from the same city and the fruit is white. Unexpectedly, these 13 accessions had a closer relationship with 'Fenhong' (A16), a cultivar with a pink fruit color. The close relationship between 'Dafudayexidi' (A24) and 'Dafuxiaoyexidi' (A25) was consistent with the morphological similarity of these two cultivars that differ only in the size of the leaves. Accessions 'Zaose' (F4) and 'Chise' (F1), which only differ in ripening date, showed a close relationship in the dendrogram as well.

Lack of agreement of the results obtained in this study with those from previous studies was noticed. 'Dongkui' (F13) and 'Biqi' (F12), the two most widely sold cultivars, clustered together in this study, like in Pan et al. (2008) and Qian et al. (2006), but was separated to two different groups as found by Xie et al. (2008) using RAPD and ISSR markers. This inconsistency might be the result of the differences in the number and geographical range of the accessions as well as the total number of amplification bands obtained as found in the individual studies. Because this study used the greatest number of accessions and obtained more than 200 amplification bands, the data presented here may be more reliable. Currently, we are applying simple sequence repeat (SSR), another molecular marker approach, in further analysis to confirm the data from this study. Thirteen SSR markers were developed from a genomic library of Chinese bayberry by Terakawa et al. (2006), but further reports on application of these markers in genetic diversity analysis were not available. Furthermore, we recently began analysis of expressed sequence tag (EST) information from four fruit cDNA libraries of 'Biqi' to develop EST-SSR markers, which will be also applied in genetic diversity analysis.

In conclusion, AFLP was successfully applied to evaluate the genetic diversity of Chinese bayberry accessions. Chinese bayberry could be clearly distinguished from wax myrtle and could be subdivided into two groups. Subgrouping of Chinese bayberry was not related to the sex of the plant and color or size of the ripe fruit, but to some extent the region where the accession originated. The obtained data established the genetic relationships of commonly cultivated Chinese bayberry accessions and which can contribute to discrimination and sorting of cultivars and lines, evaluation and use of genetic resources, and management of germplasm as well.

\section{Literature Cited}

Bao, L., K.S. Chen, D. Zhang, X.G. Li, and Y.W Teng. 2008. An assessment of genetic variability and relationships within Asian pears based on AFLP (amplified fragment length polymorphism) markers. Sci. Hort. 116:374-380.

Chen, K.S., C.J. Xu, B. Zhang, and I.B. Ferguson. 2004. Red bayberry: Botany and horticulture. Hort. Rev. 30:83-114.

Chen, K.S., C.J. Xu, B. Zhang, and I.B. Ferguson. 2008. Myrica rubra (red bayberry), p. 522-526. In: Janick, J. and R.E. Paull (eds.). The encyclopedia of fruit and nuts. Cambridge University Press, Cambridge, UK.

Handa, T. and I. Kajiura. 1991. Isozyme analysis of yamamomo (Myrica rubra Sieb. et Zucc.) cultivars. Jpn. J. Breed. 41:203-209.

Heidi, M.M. and C.C. Andrew. 2007. Almost forgotten or latest practice? AFLP applications, analyses and advances. Trend. Plant Sci. 12: 106-117.

Joyce, D.C. 2007. Evaluation of fresh red bayberry (Myrica rubra) fruit acceptance. N. Z. J. Crop Hort. Sci. 35:125-128.

Karp, D. 2007. From China, only in a bottle, a berry with an alluring name The New York Times, 12 Dec. 2007

Karp, D. 2008. Myrica rubra, a fruit of many names. Fruit Gardener 40:30-33.

Lin, B.N., L.J. Xu, and C.L. Jia. 1999. Studies on identification and classification of genomic DNA in Myrica by RAPD analysis. Acta Hort. Sin. 26:221-226. [in Chinese]

Pan, H., X.H. He, Y.W. Li, Y.Z. Guo, and G.X. Huang. 2008. Genetic diversity of wild Myrica resources in Guangxi analyzed by inter-simple sequence repeats (ISSRs). J. Fruit Sci. 25:353357. [in Chinese]

Qian, J.L., W.S. Yu, H.K. Wang, X.M. Lou, and Z Zhang. 2006. Analysis on ISSR marker of primary cultivars of bayberry in Jiangsu and Zhejiang. J. Plant Resour Environ. 15:17-20. [in Chinese].

Qiu, Y.X., C.X. Fu, and H.H. Kong. 2002. Intersimple sequence repeat (ISSR) analysis of different cultivars in Myrica rubra. J. Agr. Biotech. Sin. 10:343-346. [in Chinese].

Terakawa, M., S. Kikuchi, S. Kanetani, K. Matsui, T. Yumoto, and H. Yoshimaru. 2006. Characterization of 13 polymorphic microsatellite loci for an evergreen tree, Myrica rubra. Mol. Ecol. Notes 6:709-711.

Vos, P., R. Hogers, and M. Bleeker. 1995. AFLP: A new technique for DNA fingerprinting. Nucleic Acids Res. 23:4407-4414.

Xie, X.B., Y.Y. Qiu, X.J. Qi, X.L. Zheng, L.J. Qiu, and Y.J. Zhang. 2008. Analysis of genetic relationship between male and female plants in Myrica rubra by RAPD and ISSR. J. Fruit Sci. 25:198-202 [in Chinese].

Zhang, W.S., X. Li, J.T. Zheng, G.Y. Wang, C.D. Sun, I.B. Ferguson, and K.S. Chen. 2008. Bioactive components and antioxidant capacity of Chinese bayberry (Myrica rubra Sieb. and Zucc.) fruit in relation to fruit maturity and postharvest storage. Eur. Food Res. Technol. 227:1091-1097.

Zhang, Y.J. and S.L. Miao. 1999. Resources of red bayberry and its utilization in China. South China Fruits 28:24-25 [in Chinese]. 